\title{
ANÁLISE ENERGÉTICA E EXERGÉRTICA DE UM CICLO TÉRMICO COM APLICAÇÃO DE CICLO RANKINE- REGENERATIVO*
}

Paulo Roberto Gomes de Sousa ${ }^{1}$ Caio Glauco Sanchez ${ }^{2}$

\begin{abstract}
Resumo
O trabalho mostra a analise de energia e exergia de uma unidade termoelétrica, esta planta consiste em um ciclo regenerativo. Serão comparados os nivéis de irreversibilidade dos componentes que compõem a unidade. A escolha do ciclo regenerativo com a referência em análise, é devido sua eficiência global. Isso é proporcionado através do aquecimento da água de alimentação da caldeira, com isso, consequentemente ocorre uma diminuição da necessidade de energia imposta na caldeira. A análise realizada também mostra as irreversibilidades da planta através da análise de exergia.
\end{abstract}

Palavras-chave: Energia; Exergia; Plantas; Ciclos; Vapor; Potência.

\section{ANALYSIS OF ENERGY AND EXERGERTIC CYCLE WITH APPLICATION OF THERMAL REGENERATIVE RANKINE CYCLE}

\section{Abstract}

The work shows the energy and exergy analysis of a thermoelectric unit, this plant consists of a regenerative cycle. Will be compared levels of irreversibility of the components that make up the unit. The choice of the regenerative cycle with the reference in question is due to its overall efficiency. This is provided by heating the boiler feed water, thus consequently there is a decreased need for energy imposed in the boiler. The analysis also shows the irreversibility of the plant through exergy analysis.

Keywords: Energy; Exergy; Plant; Cycles; Steam power.

1 Engenharia/Pós-Engenharia de Manutenção e Engenharia Mecatrônica, Engenheiro/Engenheiro de Projetos, Engenharia/Desenvolvimento, TGMTurbinas, Sertãozinho, São Paulo e Brasil.

2 Professor/Pesquisador, Pós-Doutorado, Professor e Pesquisador, Departamento de Engenharia Térmica e de Fluidos, UNICAMP-Universidade Estadual de Campinas, Campinas, São Paulo, Brasil. 


\section{INTRODUÇÃO}

O ciclo de Rankine é o ciclo mais difundido a nível mundial, sendo utilizado desde o final do século XIX, este ciclo foi proposto por W.J.Rankine, físico e engenheiro escocês e por R. Clausius, físico alemão, quase simultaneamente, por volta de 1850 . O rendimento térmico deste ciclo depende das temperaturas médias em que o vapor é fornecido e rejeitado, conforme Van Wylen, (1978). O rendimento do ciclo aumenta desde que haja aumento da temperatura na qual o vapor é fornecido, ou que haja diminuição da temperatura do vapor rejeitado. Na figura 1, mostra o ciclo de Rankine simples.

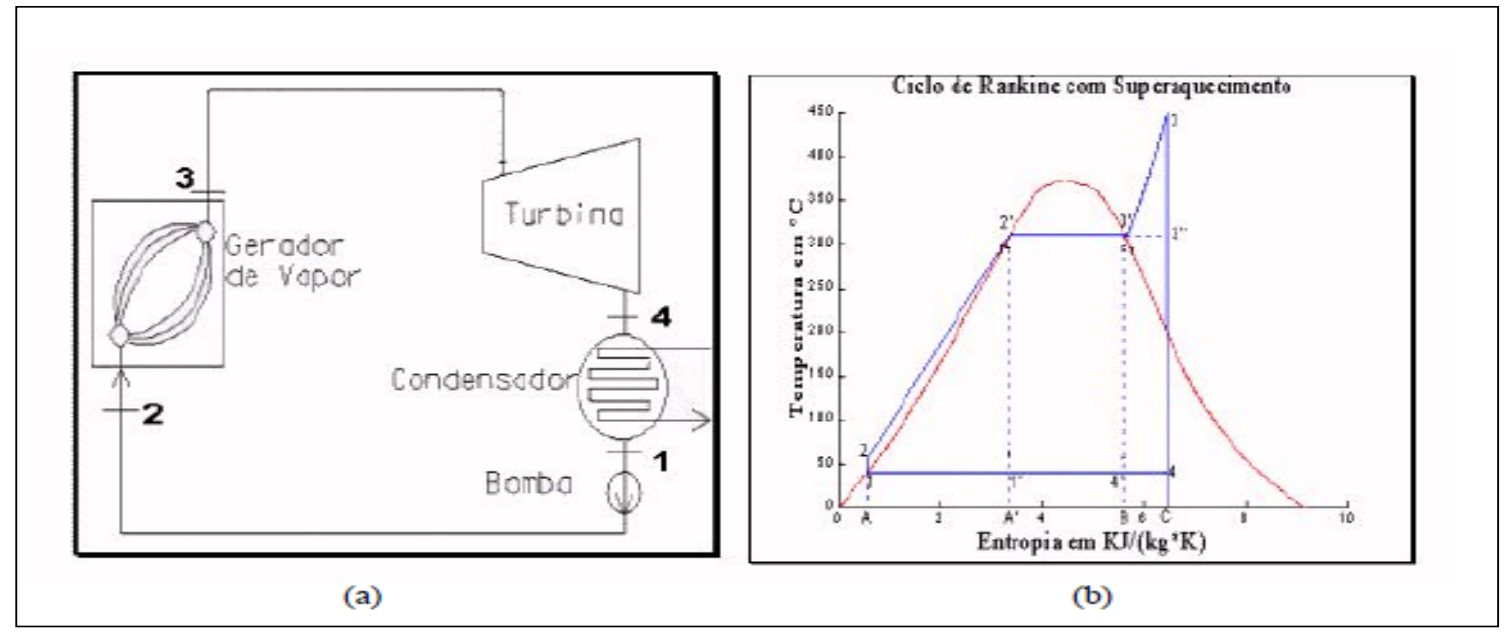

Figura 1 - Ciclo de Rankine.

A aplicação atualmente realizada tem o objetivo a eficiência global de uma planta com o ciclo regenerativo, conforme a figura 2. O ciclo regenerativo proporciona 0 aquecimento da água de alimentação da caldeira, a água é submetida a um acréscimo de energia através do aquecimento realizado pelas sangrias da turbina. Com isso, diminui-se o consumo de combustível necessário e resulta em melhores eficiências globais.

O ciclo regenerativo apresentado na figura 2 é uma variação do ciclo Rankine simples no qual a diferença principal está na presença de pré-aquecedores de água de alimentação do gerador de vapor. Esses pré aquecedores, são alimentados com vapor à determinadas condições através de sangrias realizadas na turbina que permiti diminuir o calor adicionado ao ciclo, aumentando a temperatura da água de alimentação. Porém, tal uso diminui o trabalho da turbina. A quantidade de trocadores de calor aplicado, para obter melhores eficiências globais implica em maiores custos econômicos e dificuldades na operação. Haywood (1980) e Kostyk e Frolov (1988) descreveram métodos utilizados para se determinar o número adequado de trocadores de calor para maximizar a eficiência do ciclo. 


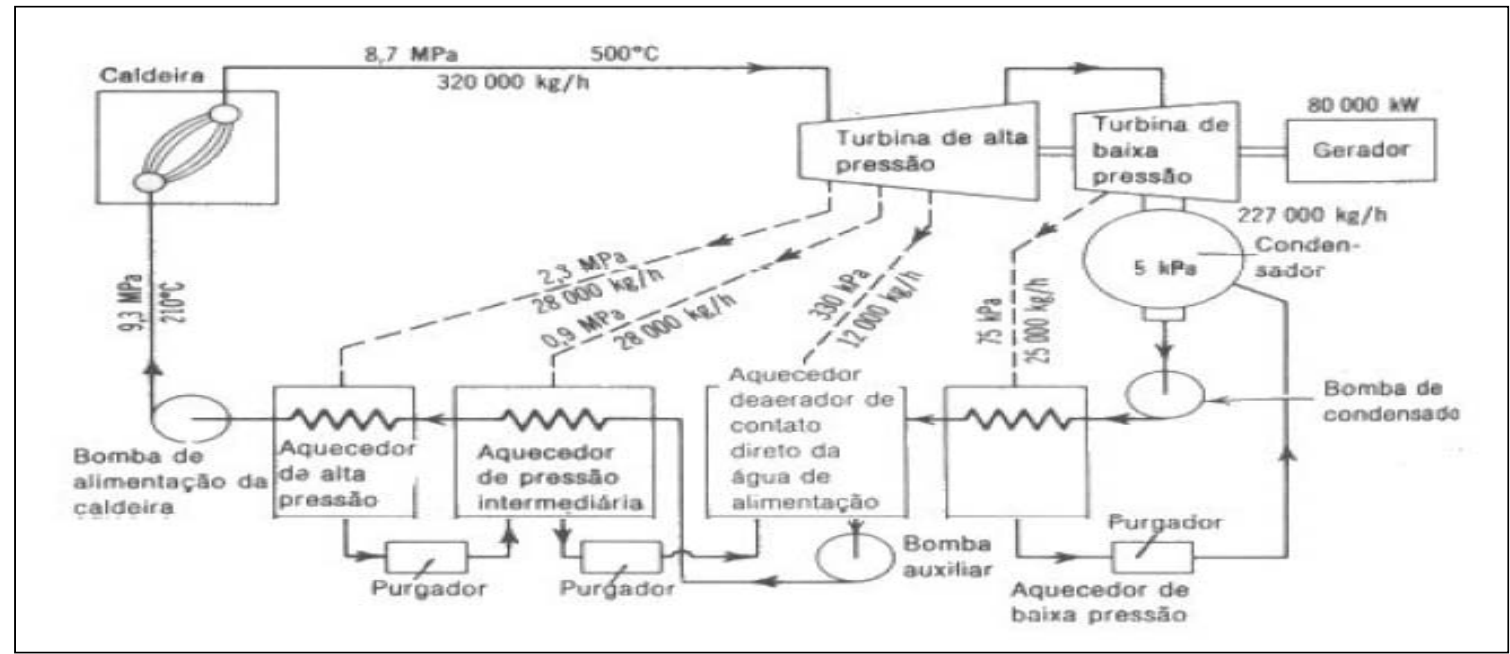

Figura 2. - Ciclo de Rankine Regenerativo.

Cada vez mais, esse ciclo está sendo empregado em todos os ramos de geração de energia. Um dos pontos positivos para que ocorra esse tipo de arranjo termodinâmico é a grande evolução dos equipamentos.

A motivação deste trabalho é o desejo de atuar no processo de desenvolvimento de metodologias e simulações de centrais termelétricas, com o intuito de contribuir positivamente ao propósito do assunto abordado.

A simulação da planta foi realizada em um programa em Excel. Possibilitando identificar a melhor aplicação possível para o ponto de melhor utilização da capacidade termodinâmica disponível, com isso, obtendo o máximo trabalho e a melhor eficiência global da planta.

Atualmente a corrida é cada vez maior em busca de melhores eficiências em todos os tipos de processos, isso visando diminuir os custos e necessidades de recursos. Com esse pensamento, obtêm-se melhores lucros e uma melhor sustentabilidade da utilização de recursos naturais. Pensando neste ponto, estão sendo projetadas caldeiras com condições de vapor em maiores faixas de trabalho que contribui com uma melhor eficiência do ciclo térmico, turbinas com melhores eficiências e com mais flexibidade operacional, isto mostra a importância da análise exergética. Com isso, obtém-se melhores análise termo econômicas de uma planta, possibilitando identificar quem são os equipamentos que mais e menos contribui para o ciclo térmico. Abaixo a figura 18 mostra a interface do programa de simulação e a tabela 1 os resultados obtidos através das simulações.

\section{MATERIAIS E MÉTODOS}

\subsection{Desenvolvimento}

Atualmente, estão sendo realizados estudos e simulações de sistemas térmicos de potência em centrais termelétricas, por serem elas contribuidoras de uma enorme fatia da geração de energia elétrica no mundo. Inúmeras modificações nos ciclos básicos são examinadas com o propósito de aumentar seus rendimentos termodinâmicos. Porque mesmo pequenos aumentos destes rendimentos podem causar um impacto sobre os recursos energéticos mundiais. Diante do enorme potencial a ser explorado e do crescente consumo de energia em todo mundo, as centrais termelétricas certamente continuarão a ser consideradas como uma das principais fontes para atender às demandas energéticas nos próximos anos. Por esta razão, é fundamental 
que os estudos e pesquisas voltadas aos sistemas térmicos avancem cada vez mais. Esses estudos de aprimoramentos dos ciclos de Rankine simples para os ciclos regenerativos é de grande importância quando o objetivo são melhores eficiências globais e melhores utilizações dos recursos naturais.

\subsection{Objetivo}

Mostrar a análise energética e exergética de uma planta térmica e o estudo de cada equipamento que constitui a unidade, tantos os equipamentos que geram trabalho e calor, quanto os equipamentos dissipativos. A eficiência exergética é a propriedade de identifica a máxima energia útil de um sistema. Com a análise exergética é possível identificar a irreversibilidade dos equipamentos em uma planta termelétrica.

Com a análise de exergia é possível verificar que a maior irreversibilidade está na caldeira e não no condensador, como é possível verificar através da análise energética.

\subsection{Modelagem dos Equipamentos}

O intuito desta seção é apresentar a modelagem dos equipamentos considerados essenciais na composição do ciclo regenerativo, que caracteriza os sistemas térmicos, que são, gerador de vapor, turbina, pré aquecedores, desaerador, condensador e bombas. Essas modelagens dos equipamentos do ciclo de regenerativo serão efetuadas a análise energética e exegética, ou seja, uma forma de comparar o comportamento da $1^{\circ}$ e $2^{\circ}$ Lei da termodinâmica.

Essas equações mostradas abaixo, são utilizadas para determinar a eficiência de cada componente que compõem o ciclo regenerativo.

A $1^{\circ}$ Lei é composta pela seguinte equação geral aplicada a um volume de controle, conforme a figura 3 .

$$
\frac{d E_{V C}}{d t}=\sum \dot{Q}_{V C}-\sum \dot{W}_{V C}+\sum_{e} \dot{m}_{e} \cdot\left(h_{e}+\frac{V_{e}^{2}}{2}+g z_{e}\right)-\sum_{s} \dot{m}_{s} .\left(h_{s}+\frac{V_{s}^{2}}{2}+g z_{s}\right)
$$

Figura $3-1^{\circ}$ Lei da termodinâmica

A $2^{\circ}$ Lei é composta pela seguinte equação geral aplicada a um volume de controle e também foi aplicado o conceito de exergia. Em um processo entre dois estados quaisquer a variação da exergia será conforme a figura 4.

$$
\begin{aligned}
& \frac{d S_{V C}}{d t}=\sum_{j} \frac{Q_{j}}{T_{j}}+\sum_{e} m_{e} s_{e}-\sum_{s} m_{s} s_{s}+\sigma_{V C} \\
& e x_{2}-e x_{1}=\left(u_{2}-u_{1}\right)+p_{0}\left(v_{2}-v_{1}\right)-T_{0}\left(s_{2}-s_{1}\right)
\end{aligned}
$$

Figura $4-2^{\circ}$ Lei da termodinâmica.

\subsubsection{Turbina}

Turbina é o equipamento responsável pela geração de potência do sistema, as formulas de análise de $1^{\circ}$ e $2^{\circ}$ Lei da termodinâmica são conforme as figuras 5 e 6 . 
$1^{\circ}$ Lei:

$$
\eta_{S T}=\frac{w_{L}}{h_{1}-h_{2 s}}=\frac{h_{1}-h_{2}}{h_{1}-h_{2 s}}
$$

Figura $5-1^{\circ}$ Lei da termodinâmica.

$2^{\circ}$ Lei:

$$
\varepsilon_{T}=\frac{P}{F}=\frac{W_{L}}{E x f_{1}-E x f_{2}}
$$

Figura $6-2^{\circ}$ Lei da termodinâmica.

\subsubsection{Gerador de Vapor}

O gerador de vapor é o equipamento onde é queimado um determinado combustível que fornece calor para água, transformando-a em vapor, ou seja, energia térmica. As figuras 7 e 8 mostra a análise de $1^{\circ}$ e $2^{\circ}$ Lei da termodinâmica aplicada no gerador de vapor (caldeira).

$1^{\circ}$ Lei:

$$
\eta_{G V}=\frac{m_{a}\left(h_{B}-h_{A}\right)}{\dot{m}_{\text {Comb }} P C I}
$$

Figura $7-1^{\circ}$ Lei da termodinâmica.

$2^{\circ}$ Lei:

$$
\varepsilon_{G V}=\frac{P}{F}=\frac{E x f_{B}-E x f_{A}}{E x f_{\text {comb }}}
$$

Figura $8-2^{\circ}$ Lei da termodinâmica.

\subsubsection{Pré Aquecedores}

Pré aquecedores é onde ocorre a troca térmica entre o vapor fornecido pela turbina e a água de alimentação do gerador de vapor (caldeira). A definição desses componentes é muito importante porque eles definem a característica da planta. As figuras 9 e 10 mostra a análise de $1^{\circ}$ e $2^{\circ}$ Lei da termodinâmica nesses equipamentos.

$$
1^{\circ} \text { Lei: } \quad \eta_{T C}=1 \quad(\text { se for adiabático })
$$

Figura $9-1^{\circ}$ Lei da termodinâmica.

$$
\text { 20 Lei: } \varepsilon_{T C}=\frac{P}{F}=\frac{E x f_{4}-E x f_{3}}{E x f_{1}-E x f_{2}}
$$

Figura $10-2^{\circ}$ Lei da termodinâmica.

\subsubsection{Bomba}

A bomba é o equipamento responsável por realizar o bombeamento e pressurização do sistema. Esse elemento consume potência para fornecer a água de alimentação nas suas devidas características, abaixo nas figuras 11 e 12, mostra como é constituído a análise de $1^{\circ}$ e $2^{\circ}$ Lei da termodinâmica.

$1^{\circ}$ Lei:

$$
\eta_{\text {SC ou } B}=\frac{h_{2 s}-h_{1}}{\left|w_{L}\right|}=\frac{h_{2 s}-h_{1}}{h_{2}-h_{1}}
$$

Figura $11-1^{\circ}$ Lei da termodinâmica. 
$2^{\circ}$ Lei:

$$
\varepsilon_{\text {Сои В }}=\frac{P}{F}=\frac{E x f_{2}-E x f_{1}}{W_{L}}
$$

Figura $12-2^{\circ}$ Lei da termodinâmica.

\subsubsection{Condensador}

Condensador é responsável por rejeitar calor do sistema. Com isso, por análise de $1^{\circ}$ Lei da termodinâmica ele é o principal responsável pela ineficiência do sistema conforme a figura 13 . Porém por análise de $2^{\circ}$ Lei da termodinâmica podemos verificar que as irreversibilidades contidas no condensador são pequenas comparadas ao da caldeira. Conforme a figura 14.

$1^{\circ}$ Lei:

$$
\eta_{C A}=\frac{Q_{\text {cond }}}{\dot{m}_{a}\left(h_{B}-h_{A}\right)+W_{B}}
$$

Figura $13-1^{\circ}$ Lei da termodinâmica.

$2^{\circ}$ Lei:

$$
\varepsilon=\frac{P}{F}=\frac{Q\left(1-\frac{T_{0}}{T}\right)}{E x f_{1}-E x f_{2}+W_{B}}
$$

Figura $14-2^{\circ}$ Lei da termodinâmica.

O ciclo é composto pela interação entre todos os componentes que resulta na análise global da planta, esse é o principal objetivo do estudo. Porque quanto maior a eficiência o ciclo, significa menores custos com recursos financeiros e naturais. Por esse motivo, a eficiência global é tão importante em uma planta térmica. As análises de $1^{\circ}$ e $2^{\circ}$ Lei da termodinâmica são aplicadas na planta conforme as figuras 15 e 16.

$$
1^{\circ} \text { Lei: } \quad \eta_{\text {ciclo }}=\frac{\text { Wliq }}{\dot{m c o m b}^{*} \text { PCI }}
$$

Figura $15-1^{\circ}$ Lei da termodinâmica.

$$
2^{\circ} \text { Lei: } \quad \eta_{\text {ciclo }}=\frac{\text { Wliq }}{\operatorname{Exf} 1^{*} \operatorname{Exf} 2}
$$

Figura $16-2^{\circ}$ Lei da termodinâmica.

\section{RESULTADOS E DISCUSSÃO}

Para mostrar o comportamento entre a análise energética e exergética, foi realizado os diagramas que representam as duas análises. O diagrama de Sankey e o diagrama de Gransmann respectivamente. Figuras 16 e 17 abaixo. 


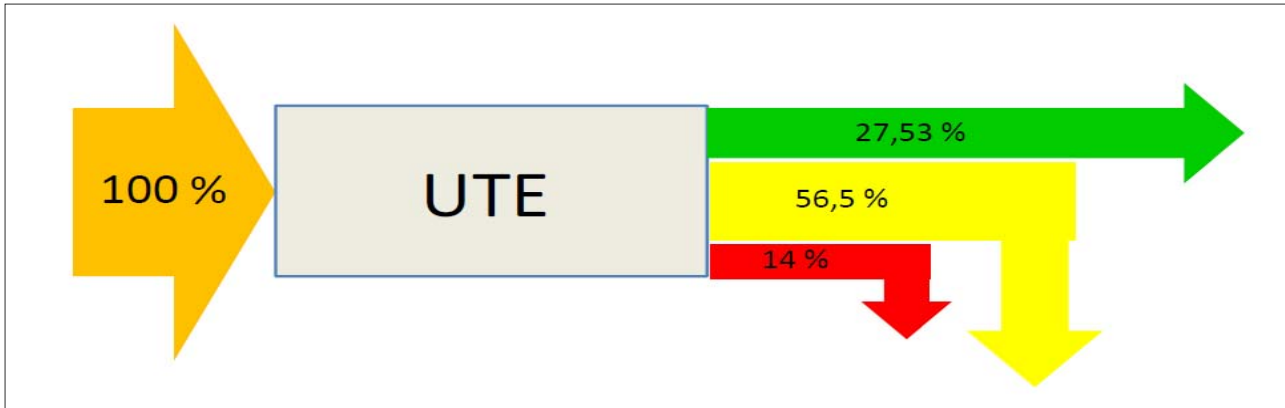

Figura 16. - Diagrama de Sankey.

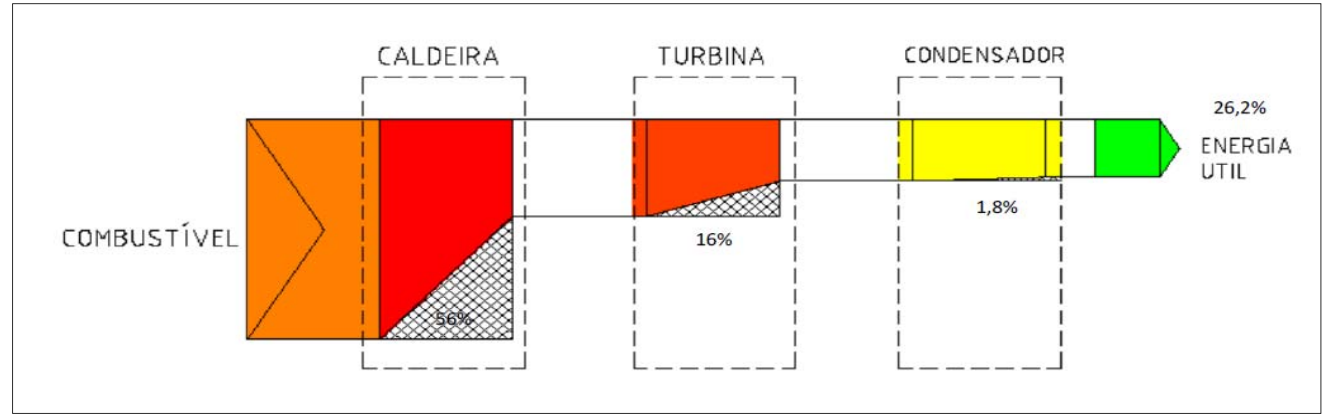

Figura 17. - Diagrama de Grassmann.

Através das simulações, foi possível identificar que a turbina é um dos principais componentes. Pelo fato, que o comportamento da turbina gera um grande efeito na planta. Foi realizado uma análise do comportamento entre a temperatura da água de alimentação da caldeira, eficiência global da planta e a potência da turbina, ou seja, os comportamentos são inversamente proporcionais, quando-se aumenta a temperatura da água de alimentação, produz menos energia, porém com um melhor desempenho energético da planta. Com isso, o sistema deixa de produzir o máximo trabalho, o comportamento das propriedades estão conforme os gráficos 1 e 2 . Então com essas análises consegue-se obter e definir o melhor ponto de operação, analisando a situação de melhor produção de trabalho útil com a melhor eficiência global do ciclo térmico. O estudo mostrou também a relação entre o comportamento da eficiência energética e exergética em relação à temperatura da água de alimentação da caldeira, conforme o gráfico 3 abaixo. Porque essa temperatura, é correspondente de uma energia que foi adicionada a água para a obtenção de um menor consumo de combustível, isso é favorável para a economia e para o meio ambiente, porque com menor consumo de combustível resulta em menor emissão de gases poluentes e menor utilização de recursos naturais.

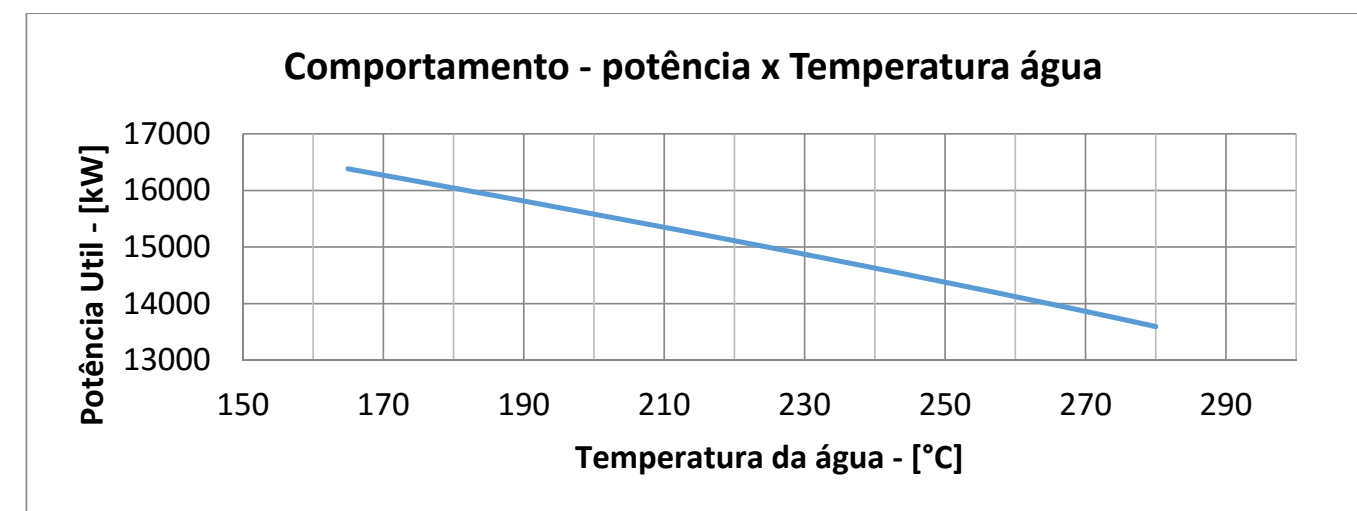

Gráfico 1. - Comportamento potência x Temperatura da água de alimentação. 


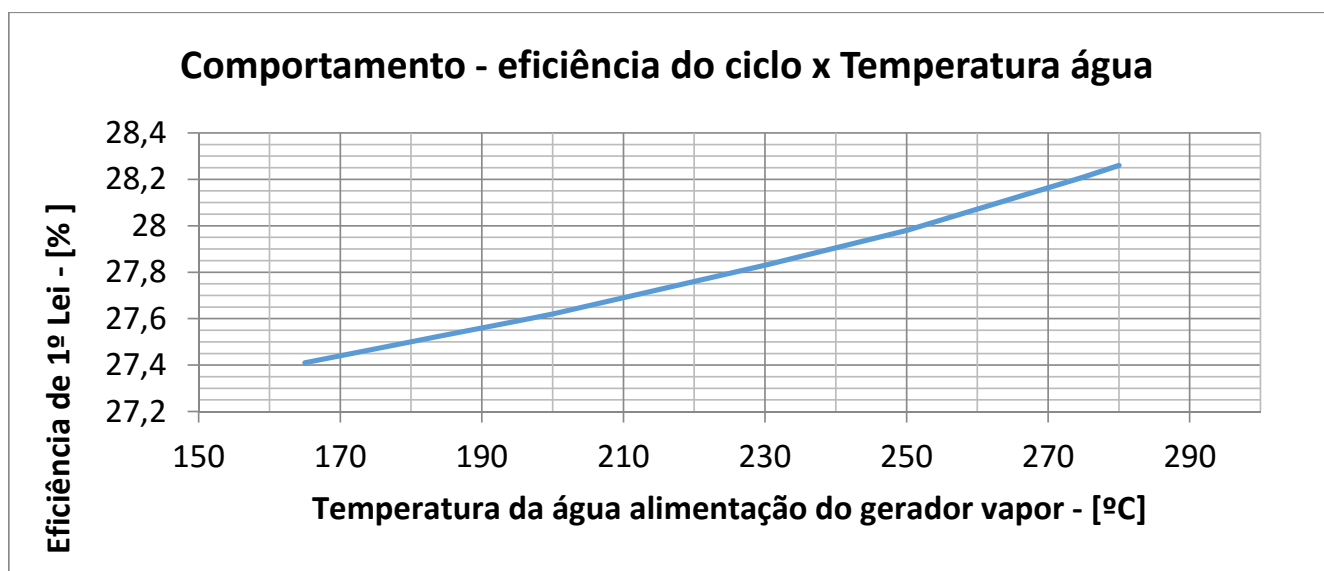

Gráfico 2. - Comportamento eficiência x Temperatura da água de alimentação.

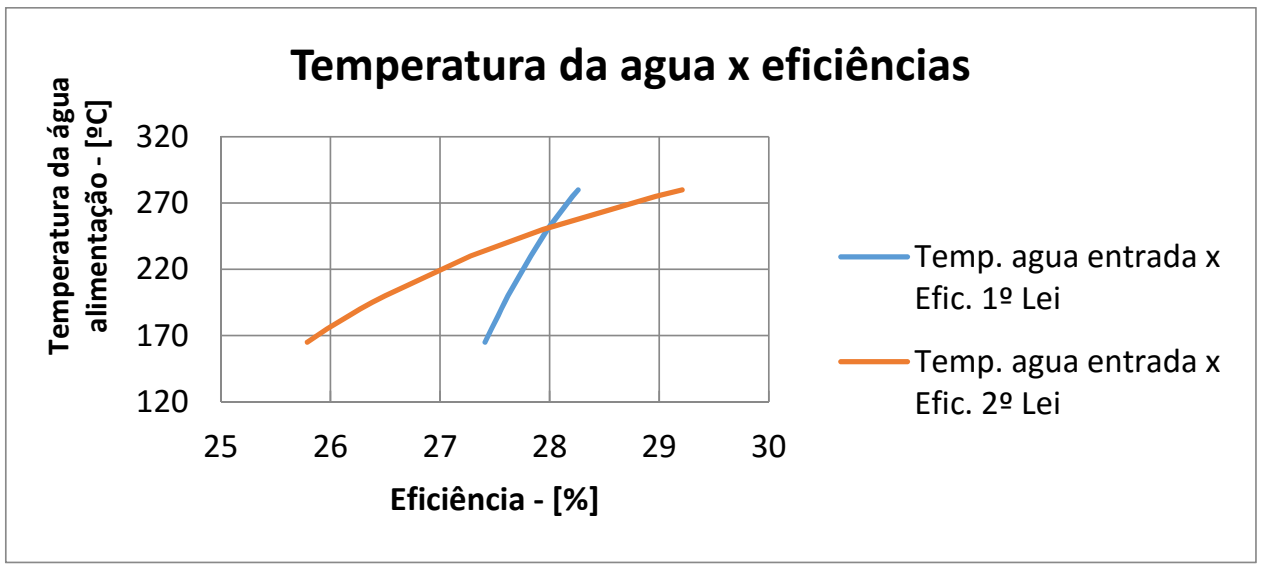

Gráfico 3. - Análise térmica através da $1^{\circ}$ e $2^{\circ}$ lei.

\section{CONCLUSÃO}

O objetivo do presente trabalho foi alcançado, devido à realização de um programa para simular um ciclo regenerativo e permitindo a comparação de análise energética e exergética de uma planta, conforme a figura 18 e a tabela 1 abaixo. Com a simulação foi observado que à eficiência exergética é menor que a energética e que a análise exergética mostra que o grande gerador de irreversibilidade de uma planta termelétrica é a caldeira e não o condensador. No gráfico 4 mostra que a potência liquida da turbina é inversamente proporcional a temperatura da água que entra na caldeira. Com essas análises é possível identificar o melhor ponto de operação da planta para otimizar a eficiência e a geração de potência. 


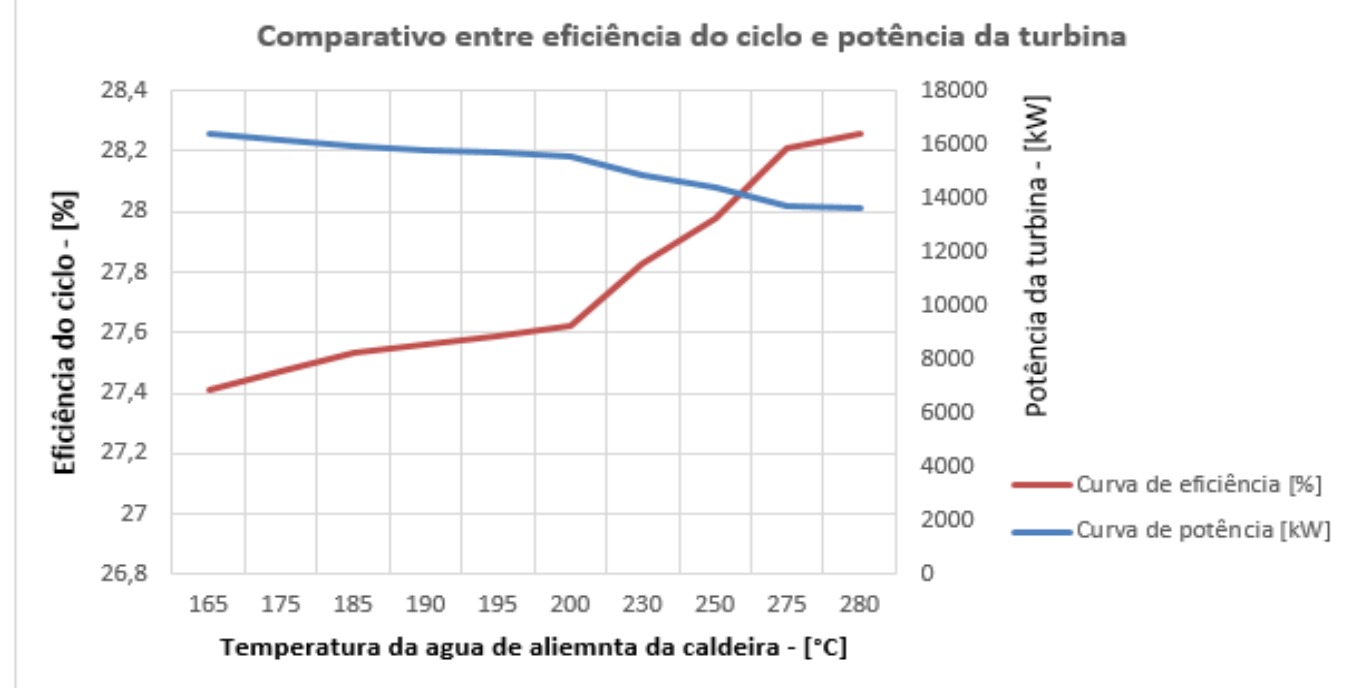

Gráfico 4. - Comparação entre eficiência do ciclo e potência da turbina.

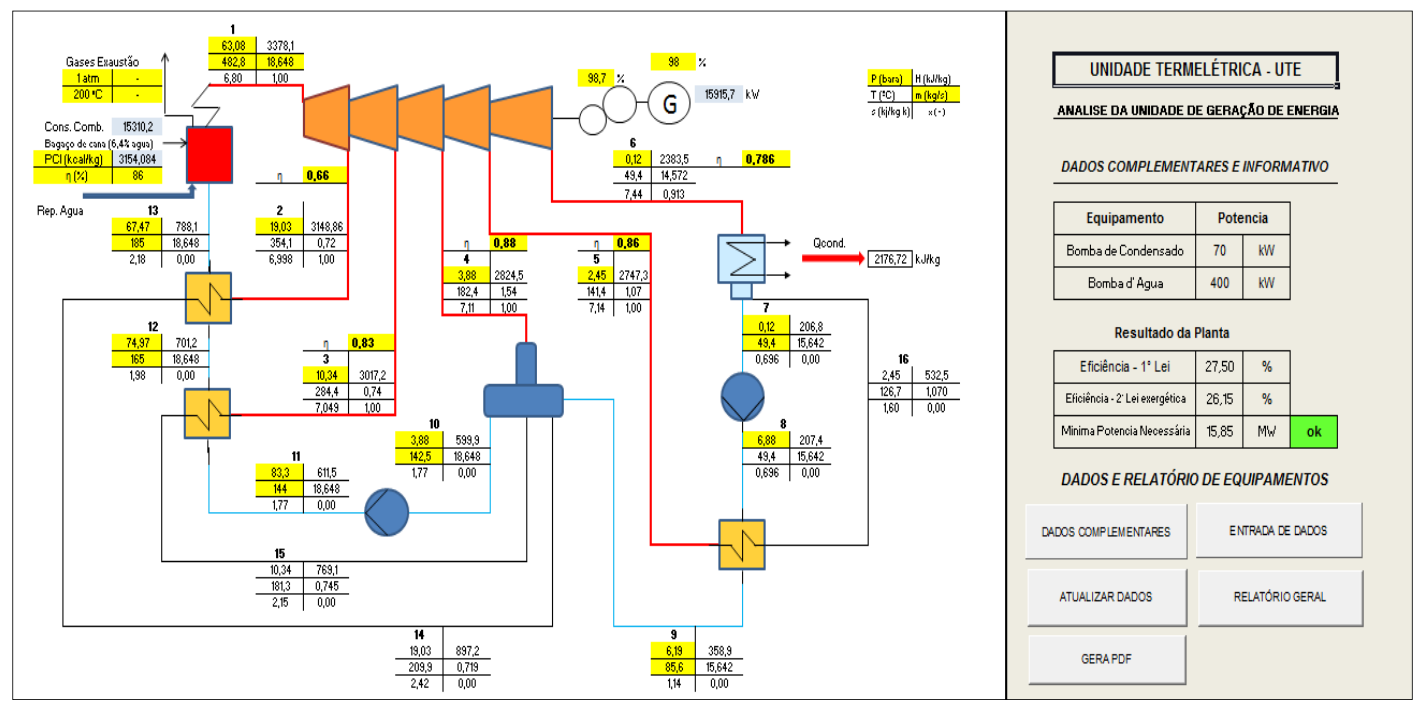

Figura 18 - Interface do programa de simulação.

\begin{tabular}{|c|c|c|c|c|c|c|}
\hline Equipamentos & $\begin{array}{c}\text { Energia } \\
\text { disponível }\end{array}$ & $\begin{array}{c}\text { Irreversibil } \\
\text { idade }\end{array}$ & $\begin{array}{c}\text { Energia } \\
\text { I útil }\end{array}$ & $\begin{array}{c}\% \\
\begin{array}{c}\text { Irreversibilid } \\
\text { ade }\end{array} \\
\end{array}$ & $\begin{array}{c}\text { Eficiência } \\
1^{\circ} \text { Lei }\end{array}$ & $\begin{array}{c}\text { Eficiência } \\
2^{\circ} \text { Lei }\end{array}$ \\
\hline Und. & kW & kW & kW & $\%$ & $\%$ & $\%$ \\
\hline Caldeira & 56160,8 & 7862,5 & 48298,3 & - & 86 & 36,5 \\
\hline Turbina total & 19589,8 & 3135,4 & 16454,4 & 1,45 & 91,12 & 83,99 \\
\hline Condensador & & 31719,4 & & 56,48 & 6,9 & 61,5 \\
\hline $\begin{array}{l}\text { Bomba } \\
\text { condensado }\end{array}$ & 70 & 53,6 & 16,4 & & - & - \\
\hline Aquecedor 3 & & 66,0 & & 0,50 & 1,0 & 3,33 \\
\hline Desaerador & & & & & 1,0 & 7,7 \\
\hline $\begin{array}{l}\text { Bomba d' } \\
\text { água }\end{array}$ & 400 & 185,1 & 214,9 & & - & - \\
\hline Aquecedor 2 & & 137,9 & & 1,04 & 1,0 & 3,36 \\
\hline Aquecedor 1 & & 185,2 & & 1,40 & 1,0 & 3,21 \\
\hline $\begin{array}{l}\text { Turbo gerador } \\
\text { TG }\end{array}$ & 18948,4 & 3135,4 & 15915,7 & - & - & - \\
\hline Planta & - & - & - & - & 27,53 & 26,2 \\
\hline
\end{tabular}

\section{Tabela 1. Equipamentos.}




\section{Agradecimentos}

Agradeço ao Prof. Dr. Eng. ${ }^{\circ}$ Caio Glauco Sanchez da Unicamp de Campinas, do departamento de Engenharia Térmica e de Fluidos, pela oportunidade de realizar o mestrado como aluno especial e a TGMTurbinas pela oportunidade de trabalhar na área térmica.

\section{REFERÊNCIAS}

1 Giancarlo Cenutti Panosso, "Métodos de simulação para ciclos de Rankine", Tese de Mestrado, Universidade Federal do Rio Grande do Sul, 2003.

2 Rodrigues, F. F., "Simulador em regime transiente de alguns equipamentos de um ciclo de Rankine regenerativo", Tese de Mestrado, Universidade Federal do Rio Grande do Sul, 2005.

3 João Gari Silva Fonseca Júnior, "Análise energética e exergética de um ciclo Rankine com aquecimento distrital", Tese de Mestrado, Universidade Federal do Rio Grande do Sul, 2003.

4 Silva, Marcelo Modesto, "Repotenciamento em sistema de geração de potência na indústria siderúrgica utilizando análise termoecomômica", Tese de Mestrado, UNICAMP, 2004.

5 Kotas, J. T., "The exergy method of thermal plant analysis", 1985.

6 Llagostera B., J.I.; Oliva G.A.; Salazar, S. "Aplicação do método da exergia à análise termodinâmica do sistema de cogeração em usinas de açúcar e álcool”. In: Anais do II Encontro Nacional de Ciências Térmicas (ENCIT), pp. 277-280, Águas de Lindóia-SP, 1988.

7 Barreda del Camp, E. R., "Avaliação das possibilidades de incremento da cogeração em usinas açucareiras cubanas", Tese de Mestrado, UNICAMP, 1995.

8 Moran, J. M. And Shapiro H. N., 2002, "Principios de Termodinâmica para Engenharia", LTC, Rio de Janeiro, Brasil.

9 Higa, M., "Cogeração e Integração Térmica em Usinas de Açúcar e Álcool", Tese de Doutorado, UNICAMP, 2003. 